\title{
Reply Comment: Comparison of Approaches to Classical Signature Change
}

\author{
Charles Hellaby \\ Department of Applied Mathematics, University of Cape Town, Rondebosch, 7r00, South Africa \\ E-mail: cwh@maths.uct.ac.za \\ $\&$ \\ Tevian Dray \\ Department of Mathematics, Oregon State University, Corvallis, Oregon 97331, USA \\ School of Physics and Chemistry, Lancaster University, Lancaster, LA1 4YB, U.K. \\ E-mail: tevian@math.orst.edu
}

Phys. Rev. D52, 7333-7339 (1995).

gr-qc/9601040

PACS numbers: $\quad 04.20 . \mathrm{Cv}, 04.20 . \mathrm{Me}, 11.30 .-\mathrm{j}$

\begin{abstract}
We contrast the two approaches to "classical" signature change used by Hayward with the one used by us (Hellaby and Dray). There is (as yet) no rigorous derivation of appropriate distributional field equations. Hayward's distributional approach is based on a postulated modified form of the field equations. We make an alternative postulate. We point out an important difference between two possible philosophies of signature change - ours is strictly classical, while Hayward's Lagrangian approach adopts what amounts to an imaginary proper "time" on one side of the signature change, as is explicitly done in quantum cosmology. We also explain why we chose to use the Darmois-Israel type junction conditions, rather than the Lichnerowicz type junction conditions favoured by Hayward. We show that the difference in results is entirely explained by the difference in philosophy (imaginary versus real Euclidean "time"), and not by the difference in approach to junction conditions (Lichnerowicz with specific coordinates versus Darmois with general coordinates).
\end{abstract}




\section{DIFFERENCES IN APPROACH}

\section{Meaning of 'classical'}

The most fundamental difference between the approach of Hayward and that of Hellaby and Dray (us) is in the treatment of the region of Euclidean signature. We (Hellaby and Dray) $[1,2]$ adopt a strictly classical view of this region, as do Ellis et al [3-7] and Dray, Manogue and Tucker [8-11], whereas Hayward [12-14], in his Lagrangian approach [12], works with squared momenta that change sign, which is equivalent to retaining the imaginary proper "time" and momentum fields that appear in the quantum cosmological treatment [see e.g. 17,18], obtaining a classical version of the quantum cosmological result. In an interesting comparison, Kerner and Martin [20-21] have done a quantum cosmological version of the strictly classical signature change, retaining the real "time" of the classical treatment of Euclidean signature, so that the wave function is oscillatory in the Euclidean region as well as the Lorentzian region. It is not surprising that these four approaches (classical or quantum cosmology with real or imaginary Euclidean "time") all lead to somewhat different results. None of them can claim to have any experimental or observational support.

\section{Appropriate form of the EFEs}

The Einstein Field equations were derived assuming a constant signature metric. For signature change, the most basic question to be addressed is that of the applicable form of the Einstein equations at a change of signature. Various postulates have been made and will be discussed below. The differences between them can be reduced to the question of whether the Euclidean proper "time" is real or imaginary. The applicable form of the conservation equations is similarly open to question.

\section{Distributional EFEs}

Formal calculation of the Einstein tensor from a step function metric results in products of distributions, so another approach is required to obtain a well defined distributional form of the Einstein field equations (EFEs). Hayward has shown $[12,14]$ that the usual form of the EFEs for a lapse function metric may be interpreted distributionally, but the derivation of these equations requires certain (unstated) smoothness assumptions. This does not in itself support those assumptions or that form of the distributional EFEs. We postulate an alternative form for the distributional EFEs based on more physical (and less restrictive) assumptions.

The approach of Kossowski and Kriele [15-16] also makes restrictive smoothness assumptions. Their comparison of two "classical" approaches investigates under what conditions they are equivalent, showing that in general they are not; and in each case under what conditions the Einstein tensor is bounded and/or continuous. However, they require the full 4-dimensional matter tensor to be not only bounded but also smooth through $\Sigma$. This means their proposal (a) is not what is done by us and by Ellis et al [3], so that their remark 2 criticising [3] and their very restrictive results from theorems 9 and 10 are not applicable.

\section{Choice of junction conditions}

The standard view for realistic Lorentzian solutions of Einstein's field equations is that the metric should be $C^{1}$ (on one or more 3 -surfaces) and piecewise $C^{3}$ (everywhere else). The surface of the moon is an example of a timelike surface dividing regions of different density. The 
time of decoupling in the early universe is an example of a spacelike surface dividing regions of different pressure. In other words, some components of the Einstein/matter tensor have jumps at junctions. How should a surface of signature change be treated? It is entirely reasonable to permit similar or geater discontinuities at a signature change - a change which is far more fundamental than a change in the equation of state.

In the Lagrangian approach [12], the arguments for the vanishing of the momenta are similar to those used in quantum cosmology $[17,18]$ for the vanishing of the extrinsic curvature. To satisfy the Einstein field equations (EFEs) and conservation equations, through the signature change surface $\Sigma$, implicitly requires the metric and the matter fields (in this case a scalar field $\phi)$ to be $C^{3}$. In contrast, the Lichnerowicz junction conditions for the case of no signature change (which are equivalent to the constant signature Darmois junction conditions) say that if you can find admissible coordinates spanning $\Sigma$ in which the 4 -metric is $C^{1}$, then you have a good matching. At a signature change, this continuity (whether $C^{1}$ or $C^{3}$ ) also requires the choice of a particular class of coordinates - lapse-shift coordinates with a squared lapse function, such as $N(t)=t$. We point out below that this unusually high degree of continuity can only be achieved in a limited sense, owing to the fact that the metric is degenerate at $\Sigma$, meaning the inverse metric is divergent.

The alternative choice of the Darmois junction conditions is attractive because they are expressed in terms of invariants of the signature change surface $\Sigma$ - the intrinsic (3-d) metric $\xi_{i j}$ and the extrinsic curvature $K_{i j}$. In the familiar constant signature case, if the one-sided limits of these two on one side of $\Sigma$ (i.e. in manifold with boundary $M^{+}$) equal the onesided limits on the other side $\left(M^{-}\right)$, then there is a good matching, and it is possible to find coordinates spanning $\Sigma$ which make the metric and the inverse metric at least $C^{1}$. The (onesided limits of these) invariants do not depend on the choice of coordinates in the enveloping manifolds $M^{-}$and $M^{+}$. In particular, for a spacelike surface, they do not depend on the choice of time or lapse function. For the case of a change of signature at $\Sigma$, careful analysis [1] showed that no modification of these conditions, or any special coordinate choice is required. The Darmois conditions are blind to the change in signature, just as the EFEs are blind to the metric signature. Of course it is no longer possible to find coordinates that make both the metric and its inverse $C^{1}$, but, as above, the covariant metric can be made arbitrarily smooth. Thus all the main results in [1] are coordinate invariant. Supplementary junction conditions on the equation of state or the matter fields may also be imposed, and such conditions were given in [1] for an electromagnetic field, as well as the consequences of the EFEs at a signature change in an arbitrary electrovac model, in equations (78)-(80).

Standard conservation can be shown to hold as a consequence of the constant signature Darmois (or Lichnerowicz) conditions. At a signature change, the same conditions lead to extra jumps in physical quantities, such as the density, and a surface term in the derived conservation

law. Conservation is not abandoned, but it has to be modified. Stronger conditions than those in [12] are required to restore the conservation law to its familiar form, for the strictly classical case.

\section{THE CASE OF A HOMOGENEOUS SCALAR FIELD}

\section{The Field Equations for a squared-lapse metric}

Consider the case of a homogenous isotropic universe with scale factor $a(t)$ and squared lapse 
function $N(t)$, filled with a scalar field $\phi(t)$ with arbitrary potential $V(\phi)$. The line element

$$
d s^{2}=-N(t) d t^{2}+{ }^{3}{ }_{i j} d y^{i} d y^{j}=-N(t) d t^{2}+a^{2}(t)\left\{\frac{d r^{2}}{\left(1-k r^{2}\right)}+r^{2}\left(d \theta^{2}+\sin ^{2} \theta d \chi^{2}\right)\right\}
$$

has $N C^{0}$ and $N(t=0)=0$ on the signature change surface $\Sigma$. The $t t$ and $r r$ EFEs $G_{\beta}^{\alpha}=\kappa T_{\beta}^{\alpha}$, evaluate to $[24]$

$$
\begin{aligned}
G_{t}^{t}=-\frac{3}{a^{2}}\left\{k+\frac{\left(a^{\prime}\right)^{2}}{N}\right\} & =-\frac{\kappa}{2}\left\{\frac{\left(\phi^{\prime}\right)^{2}}{N}+V\right\}=\kappa T_{t}^{t} \\
G_{r}^{r}=\left\{\frac{a^{\prime} N^{\prime}}{a N^{2}}-\frac{\left(2 a a^{\prime \prime}+\left(a^{\prime}\right)^{2}\right)}{a^{2} N}-\frac{k}{a^{2}}\right\} & =\frac{\kappa}{2}\left\{\frac{\left(\phi^{\prime}\right)^{2}}{N}-V\right\}=\kappa T_{r}^{r}
\end{aligned}
$$

where $^{\prime}=\partial / \partial t$, the $\theta \theta$ and $\chi \chi$ equations adding nothing new. The covariant and contravariant forms re-scale the $t t$ but not the $r r$ equations by factors of $N$. The Klein-Gordon equation is implied by the EFEs.

Strictly speaking, these equations are not well defined on $\Sigma$ except in a limiting sense as $N \rightarrow 0$, and for $k$ and $V$ non-zero, $G^{t t}=\kappa T^{t t}$ is divergent in this coordinate system, regardless of the behaviour of $a^{\prime}$ and $\phi^{\prime}$ near $N=0$. Similarly, the conservation equation $\nabla_{\alpha} G_{\beta}^{\alpha}$ or $\nabla_{\alpha} G^{\alpha \beta}$ is badly defined at $N=0$. The latter form is especially problematic, since $G^{t t}$ is divergent for $k \neq 0$. One could therefore question whether the EFEs and the conservation equation are well defined on $\Sigma$. A reasonable possibility is to evaluate these equations away from $N=0$ and rescale by factors of $N$, to obtain equations which are well defined at $N=0$. The 'second order equations' of [12] and equations (2) of [14] are essentially re-scalings by factors of $N$, of the Einstein, Raychaudhury, Klein-Gordon, and/or Friedmann equations. Note that the re-scaling itself is only well-defined in the limit as $t \rightarrow 0$, not at $t=0$, itself.

\section{The Darmois approach with real "time"}

In this approach, we postulate that two metrics of different signature, each of which solves the respective constant signature form of the EFEs, may be pieced together so that the Darmois junction conditions are satisfied at the signature change surface $\Sigma$. Even if the EFEs are well defined at $\Sigma$ in each solution, the resulting matter fields are not necessarily smooth when put together. This is true with or without signature change.

We patch together the Lorentzian and Euclidean signature forms of the above metric (11) $\left(M^{-}\right.$and $\left.M^{+}\right)$, identifying the two $t=0$ surfaces in each to form the junction surface $\Sigma$. Using the notation of [1], the strictly classical treatment requires a real proper "time"

$$
d \tau=\sqrt{-\epsilon N} d t, \quad \epsilon=\left\{\begin{array}{rll}
+1, \quad t \leq 0, & N \leq 0 & \text { Euclidean signature metric } \\
-1, \quad t \geq 0, & N \geq 0 & \text { Lorentzian signature metric }
\end{array}\right.
$$

The choice of the Darmois approach, in which all quantities are well defined without re-scaling, in the limiting sense given in [1], is made largely because of this extra measure of discontinuity, and partly because of the above-mentioned singularities in the metric and Einstein tensors. In $M^{ \pm}$we calculate

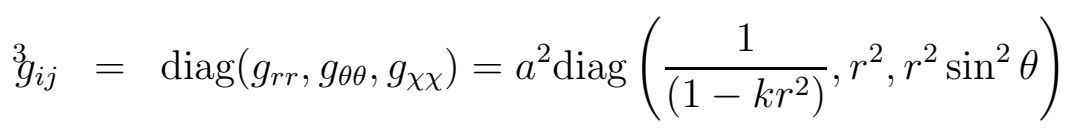




$$
\begin{aligned}
n^{\alpha} & =\left(\frac{1}{\sqrt{-\epsilon N}}, 0,0,0\right), \quad n_{\alpha} n^{\alpha}=\epsilon \\
l_{\alpha} & =(\sqrt{-\epsilon N}, 0,0,0), \quad l_{\alpha} l^{\alpha}=\epsilon \\
K_{i j} & =\operatorname{diag}\left(K_{r r}, K_{\theta \theta}, K_{\chi \chi}\right)=\frac{a a^{\prime}}{\sqrt{-\epsilon N}} \operatorname{diag}\left(\frac{1}{\left(1-k r^{2}\right)}, r^{2}, r^{2} \sin ^{2} \theta\right) \\
K & =\frac{3 a^{\prime}}{a \sqrt{-\epsilon N}}=3\left(\frac{d(\ln a)}{d \tau}\right) \\
{ }^{3} R & =\frac{6 k}{a^{2}} \\
{ }^{3} \nabla_{j} K^{i j} & =0 \\
{ }^{3} \nabla_{i} K & =0 \\
\tilde{T}^{00} & =T^{\mu \nu} l_{\mu} l_{\nu}=\epsilon \tilde{T}_{0}^{0}=\frac{-\epsilon}{2}\left\{\frac{\left(\phi^{\prime}\right)^{2}}{N}+V\right\}=\frac{1}{2}\left\{\left(\frac{d \phi}{d \tau}\right)^{2}-\epsilon V\right\} \\
\frac{a^{2}}{\left(1-k r^{2}\right)} \tilde{T}^{01} & =\tilde{T}_{1}^{0}=T_{\nu}^{\mu} l_{\mu} e_{1}^{\nu}=0
\end{aligned}
$$

where $n^{\alpha}$ and $l_{\alpha}$ are chosen to point from $M^{-}$into $M^{+}$. The tilde indicates a quantity evaluated in geodesic normal coordinates based on $\Sigma$, used for the purpose of easy interpretation, and for compact notation indicating invariants. With the notation

$$
[Z]=\left.Z\right|_{\Sigma} ^{+}-\left.Z\right|_{\Sigma} ^{-},\left.\quad Z\right|_{\Sigma}=\operatorname{Lim}_{\rightarrow \Sigma} Z
$$

for the jump in the value of $Z$ across $\Sigma$, and choosing to keep $r, \theta$ and $\phi$ continuous through $\Sigma$, the first Darmois conditions $\left[3_{i j}\right]=0$ give the obvious relations

$$
k^{+}-k^{-}=[k]=0, \quad a_{\Sigma}^{+}-a_{\Sigma}^{-}=[a]=0
$$

and the second Darmois conditions $\left[K_{i j}\right]=0$ give

$$
\left.\frac{a^{\prime}}{\sqrt{-\epsilon N}}\right|_{\Sigma} ^{+}-\left.\frac{a^{\prime}}{\sqrt{-\epsilon N}}\right|_{\Sigma} ^{-}=\left[\frac{a^{\prime}}{\sqrt{-\epsilon N}}\right]=0=\left.\frac{d a}{d \tau}\right|_{\Sigma} ^{+}-\left.\frac{d a}{d \tau}\right|_{\Sigma} ^{-}=\left[\frac{d a}{d \tau}\right]
$$

where $\tau$ is the comoving proper "time". Appropriate supplementary junction conditions for the scalar field are

$$
[\phi]=0, \quad\left[n^{\alpha} \partial_{\alpha} \phi\right]=\left[\frac{d \phi}{d \tau}\right]=\left[\frac{\phi^{\prime}}{\sqrt{-\epsilon N}}\right]=0, \quad[V]=0, \quad\left[\frac{d V}{d \phi}\right]=0
$$

From eqs (47)-(50) of [1], the various surface effects in the conservation law are then

$$
\begin{aligned}
E\left(l_{\alpha}\right)=\left[\tilde{G}^{00}\right]=-{ }^{3} R & =-\frac{6 k}{a_{\Sigma}^{2}}=\kappa\left[\tilde{T}^{00}\right]=-\kappa V_{\Sigma} \\
E\left(\bar{e}_{\alpha}^{1}\right)=\left[\tilde{G}^{01}\right]=2\left({ }^{3} \nabla_{j} K^{1 j}-{ }^{3} g^{1 j} \nabla_{j} K\right) & =0=\kappa\left[\tilde{T}^{01}\right]=0 \\
E\left(n^{\alpha}\right)=\left[\tilde{G}_{0}^{0}\right]=K^{2}-K_{i j} K^{i j} & =6\left(\frac{\left(a^{\prime}\right)^{2}}{-\epsilon N a^{2}}\right)_{\Sigma}=\kappa\left[\tilde{T}_{0}^{0}\right]=\kappa\left(\frac{\left(\phi^{\prime}\right)^{2}}{-\epsilon N}\right)_{\Sigma} \\
E\left(\bar{e}_{1}^{\alpha}\right)=\left[\tilde{G}_{1}^{0}\right]=2\left({ }^{3} \nabla_{j} K_{1}^{j}-{ }^{3} \nabla_{1} K\right) & =0=\kappa\left[\tilde{T}_{1}^{0}\right]=0
\end{aligned}
$$


Assuming that $\Sigma$ is not already a singular surface in one or both of $M^{-}$or $M^{+}$separately, these quantities are all well defined in the limit as $N \rightarrow 0$ without re-scaling. From the EFEs we obtain the restrictions

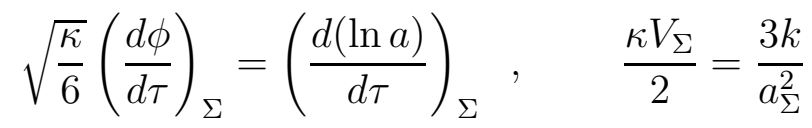

and while $(d a / d \tau)_{\Sigma}=0$ and $(d \phi / d \tau)_{\Sigma}=0$ are possibilites, they are not demanded. In any case it is not possible to remove the surface effect $E\left(l_{\alpha}\right)=-\left.{ }^{3} R\right|_{\Sigma}=-\left(6 k / a_{\Sigma}^{2}\right)$ for $k \neq 0$, in this scenario.

In summary, we are given $a$ and $\phi$ of the form

$$
a=a_{+}(t) \Theta(t)+a_{-}(t) \Theta(-t), \quad \phi=\phi_{+}(t) \Theta(t)+\phi_{-}(t) \Theta(-t)
$$

such that $a_{ \pm}, \phi_{ \pm} \in C^{3}$ separately solve the constant signature EFEs, as well as satisfying the Darmois and Klein-Gordon field junction conditions (16)-(18) and signature change condition (23). We also assume

$$
\lim _{t \rightarrow 0} \frac{a_{ \pm}^{\prime}}{\sqrt{-\epsilon N}}, \quad \lim _{t \rightarrow 0} \frac{\phi_{ \pm}^{\prime}}{\sqrt{-\epsilon N}} \quad \text { and } \quad \lim _{t \rightarrow 0}\left(\frac{2 a_{ \pm}^{\prime \prime}}{N}-\frac{a_{ \pm}^{\prime} N^{\prime}}{N^{2}}\right) \quad \text { all exist. }
$$

It follows from (16) and (18) that $[a]=0=[\phi]$, so

$$
\begin{aligned}
a^{\prime} & =a_{+}^{\prime}(t) \Theta(t)+a_{-}^{\prime}(t) \Theta(-t)+[a] \delta(t)=a_{+}^{\prime}(t) \Theta(t)+a_{-}^{\prime}(t) \Theta(-t) \\
\phi^{\prime} & =\phi_{+}^{\prime}(t) \Theta(t)+\phi_{-}^{\prime}(t) \Theta(-t)+[\phi] \delta(t)=\phi_{+}^{\prime}(t) \Theta(t)+\phi_{-}^{\prime}(t) \Theta(-t) \\
a^{\prime \prime} & =a_{+}^{\prime \prime}(t) \Theta(t)+a_{-}^{\prime \prime}(t) \Theta(-t)+\left[a^{\prime}\right] \delta(t)
\end{aligned}
$$

While condition (17), $0=[\dot{a}]=\left[a^{\prime}\right] / \sqrt{|N|}$, certainly ensures $\left[a^{\prime}\right]=0$, we must verify that it is sufficient to eliminate distributions when we insert this into (2) and (3). For example, putting $\equiv d / d \tau$,

$$
\begin{aligned}
{[\dot{a}]=0=[a] \quad \Rightarrow \quad \ddot{a} } & =\ddot{a}_{+} \Theta(t)+\ddot{a}_{-} \Theta(-t) \\
& =\left(-\frac{N^{\prime} a_{+}^{\prime}}{2 N^{2}}+\frac{a_{+}^{\prime \prime}}{N}\right) \Theta(t)+\left(\frac{N^{\prime} a_{-}^{\prime}}{2 N^{2}}-\frac{a_{-}^{\prime \prime}}{N}\right) \Theta(-t) \\
\Rightarrow \epsilon \ddot{a} & =\left(\frac{N^{\prime} a_{+}^{\prime}}{2 N^{2}}-\frac{a_{+}^{\prime \prime}}{N}\right) \Theta(t)+\left(\frac{N^{\prime} a_{-}^{\prime}}{2 N^{2}}-\frac{a_{-}^{\prime \prime}}{N}\right) \Theta(-t) \\
& =\left(\frac{N^{\prime} a^{\prime}}{2 N^{2}}-\frac{a^{\prime \prime}}{N}\right)
\end{aligned}
$$

and there is thus no distribution in $a^{\prime \prime} / N$. Conversely, if $\left(\left(N^{\prime} a^{\prime}\right) /\left(2 N^{2}\right)-a^{\prime \prime} / N\right)$ is free of distributions, then $\epsilon \ddot{a}$ is. Therefore all terms in the EFEs are bounded, and we have a solution of (2) and (3), as well as the Klein-Gordon equation. We stress that the Darmois conditions are imposed on $a(t), \phi(t)$ and $V(\phi)$ before calculating $G_{\nu}^{\mu}$ and $T_{\nu}^{\mu}$ from them. Our assumptions are borne out by solutions to (22)-(3) which will be exhibited elsewhere [27]. A rigorous treatment of distributions in the presence of signature change will appear in [26].

\section{The distributional EFEs}

In the distributional approach, we must postulate a form of the EFEs that makes sense when $N(t)$ becomes the the step function $-\Theta(-t)+\Theta(t)=\varepsilon(t)$ and $N^{\prime}$ becomes $2 \delta$, where 
$\Theta(t)$ is the Heaviside function and $\delta(t)$ is the Dirac delta function. In particular products of distributions must be avoided. Then $t=\tau$ and $\varepsilon=-\epsilon$. There is currently no known way to derive such equations from first principles.

Postulate A. The re-scaled EFEs, $N \times(2)$ and $N^{2} \times(3)$, are such a form if it is assumed that $a, a^{\prime}, \phi, \phi^{\prime}, V(\phi)$ (and $d V / d \phi$ ) are at least $C^{0}$, and $a^{\prime \prime}$ (and $\phi^{\prime \prime}$ ) may contain a step function, but no distribution. It then follows $[12,14]$ that there is a distributional term in $N^{2} \times G_{r}^{r}$, but not in $N^{2} \times \kappa T_{r}^{r}$, unless $a_{\Sigma}^{\prime}=0$. Given these assumptions with this postulated form of the distributional EFEs, $a_{\Sigma}^{\prime}=0$ is required.

However, inspection of $a^{\prime \prime}$ in (31) and in solutions to (2)-(3) [27], with $N \rightarrow \varepsilon$ and $N^{\prime} \rightarrow 2 \delta$, suggests that this assumption may be too strong, and makes it questionable that $a^{\prime \prime} \rightarrow\left(d^{2} a / d \tau^{2}\right)$ without any extra distributional terms. In other words, the distributional term that appears in $N^{2} \times(3)$ when $N^{\prime} \rightarrow \delta$ is only present because (22)-(3) were derived and re-scaled using the differentiable structure of a continuous $N$, which is not appropriate for a step function $\varepsilon$.

Postulate B. Recognising that this substitution effectively replaces lapse "time" with proper "time", $t \rightarrow \tau$, which affects the functional form of $a$, we consider it safer to transform from $t$ to $\tau \neq t$, rather than substituting functions with distributions. We write ${ }^{\circ} \equiv d / d \tau,{ }^{\prime} \equiv d^{2} / d \tau^{2}$ and we continue to assume the Darmois and scalar field junction conditions, (16)-(18), to eliminate any distributions in the following transformations:

$$
\begin{gathered}
\tau(t)=\left\{\begin{array}{l}
\tau_{+}=\int_{0}^{t} \sqrt{N(t)} d t, \quad t \geq 0 \\
\tau_{-}=-\int_{t}^{0} \sqrt{-N(t)} d t, \quad t \leq 0
\end{array}\right. \\
a=a(\tau(t)), \quad a^{\prime}=\left\{\begin{array}{ll}
\sqrt{N} \dot{a}, & t \geq 0 \\
\sqrt{-N} \dot{a}, & t \leq 0
\end{array} \quad, \quad a^{\prime \prime}=\left\{\begin{array}{l}
N \ddot{a}+\frac{N^{\prime}}{2 \sqrt{N}} \dot{a}, t \geq 0 \\
-N \ddot{a}-\frac{N^{\prime}}{2 \sqrt{-N}} \dot{a}, \quad t \leq 0
\end{array}\right.\right. \\
\phi=\phi(\tau(t)), \quad \phi^{\prime}= \begin{cases}\sqrt{N} \dot{\phi}, & t \geq 0 \\
\sqrt{-N} \dot{\phi}, & t \leq 0\end{cases}
\end{gathered}
$$

Using these expressions in (26)-(28) and substituting the result into (26) and (3) leads to:

$$
\begin{aligned}
G_{t}^{t}=-\frac{3}{a^{2}}\left\{k-\epsilon(\dot{a})^{2}\right\} & =-\frac{\kappa}{2}\left\{-\epsilon(\dot{\phi})^{2}+V\right\}=\kappa T_{t}^{t} \\
G_{r}^{r}=\left\{\epsilon \frac{\left(2 a \ddot{a}+(\dot{a})^{2}\right)}{a^{2}}-\frac{k}{a^{2}}\right\} & =\frac{\kappa}{2}\left\{-\epsilon(\dot{\phi})^{2}-V\right\}=\kappa T_{r}^{r}
\end{aligned}
$$

where we have again used (29). We note that this form of the equations does not contain $N$ or $N^{\prime}$, nor does it need rescaling, so it seems more natural for a proper time solution. We have assumed that $a, \dot{a}, \phi, \dot{\phi}, V(\phi)$, (and $d V / d \phi)$ are at least $C^{0}$, so that $\ddot{a}$ (and $\ddot{\phi}$ ) may contain a step function but no distribution, and we postulate (33) and (34) for the field equations when $N \rightarrow \varepsilon$. All of the solutions in [27] satisfy these EFEs. In particular, $\dot{a}_{\Sigma}=0$ is not required.

\section{The Lagrangian approach with imaginary "time"}

We postulate a Lagrangian approach in which the squared momenta are required to be smooth. The action for this case, in terms of the gravitational and Klein-Gordon Lagrangian 
densities can be written as

$$
\begin{aligned}
S & =\int\left(\mathcal{L}_{G}+2 \kappa \mathcal{L}_{K G}\right) d^{4} x \\
\mathcal{L}_{G} & =\sqrt{-g} R=\sqrt{\xi} N\left({ }^{3} R+K_{i j} K^{i j}-K^{2}\right) \\
\mathcal{L}_{K G} & =-\frac{\sqrt{-g}}{2}\left\{\left(\partial_{\mu} \phi\right) g^{\mu \nu}\left(\partial_{\nu} \phi\right)+V(\phi)\right\}
\end{aligned}
$$

where $R$ and $g$ are the Ricci scalar and the metric determinant, and $3=a^{6} r^{4} \sin ^{2} \theta /\left(1-k r^{2}\right)$ is the determinant of the 3 -metric ${ }^{3} y_{i j}$ of (1). Leaving the 4 -volume element $\sqrt{-g} d^{4} x$ in this form leads to an effective imaginary proper "time"

$$
d \sigma=\sqrt{N} d t
$$

in the Euclidean region, $N<0$. This leads to an imaginary unit "normal" $\hat{n}^{\alpha}=d x^{\alpha} / d \sigma=$ $(1 / \sqrt{N}, 0,0,0)$ for $N<0$ which still obeys $\hat{n}^{\alpha} \hat{n}_{\alpha}=-1$. In this way this region is not Euclidean in the classical sense. It also means the extrinsic curvature and the momenta change from imaginary to real across $N=0$, as noted by Hayward [12],

$$
\begin{aligned}
\psi & =\frac{\partial \mathcal{L}}{\partial \phi^{\prime}}=\hat{n}^{\alpha} \partial_{\alpha} \phi=\frac{\phi^{\prime} a^{3} r^{2} \sin \theta}{\sqrt{N\left(1-k r^{2}\right)}} \\
K_{i j} & =\hat{n}^{\alpha} \nabla_{\alpha}{ }^{3} g_{i j}=\frac{3_{i j}^{\prime}}{\sqrt{N}} \\
p_{j}^{i} & ={ }^{3} g_{j k} \frac{\partial \mathcal{L}}{\partial{ }^{3} g_{i k}^{\prime}}=\sqrt{3}\left(K_{j}^{i}-K \delta_{j}^{i}\right), \quad K=K_{m}^{m} \\
& =-\frac{2 a^{2} a^{\prime} r^{2} \sin \theta}{a \sqrt{N\left(1-k r^{2}\right)}} \delta_{j}^{i}
\end{aligned}
$$

and the squared momenta change sign,

$$
\begin{aligned}
\psi^{*} & =\psi^{2}=\frac{\left(\phi^{\prime}\right)^{2} a^{6} r^{4} \sin ^{2} \theta}{N\left(1-k r^{2}\right)} \\
\left(p^{*}\right)_{k}^{i} & =p_{j}^{i} p_{k}^{j}=K_{j}^{i} K_{k}^{j}-2 K K_{k}^{i}+K^{2} \delta_{k}^{i} \\
& =\frac{4\left(a^{\prime}\right)^{2} a^{4} r^{4} \sin ^{2} \theta}{N\left(1-k r^{2}\right)} \delta_{k}^{i}
\end{aligned}
$$

implying $\psi^{*},\left(p^{*}\right)^{i j}, \psi, p^{i j}$ and $K_{i j}$ must all be zero at the change surface assuming the momenta are required to be smooth and $a, \phi, \phi^{\prime}$ and $a^{\prime}$ all real.

The Lagrangian approach with real "time"

One could argue that it is more reasonable to define the action using a real volume element $\sqrt{|g|} d^{4} x$

$$
\begin{aligned}
S & =\int\left(\mathcal{L}_{G}+2 \kappa \mathcal{L}_{K G}\right) d^{4} x \\
\mathcal{L}_{G} & =\sqrt{|g|} R=\sqrt{3|N|}\left({ }^{3} R+K_{i j} K^{i j}-K^{2}\right) \\
\mathcal{L}_{K G} & =-\frac{\sqrt{|g|}}{2}\left\{\left(\partial_{\mu} \phi\right) g^{\mu \nu}\left(\partial_{\nu} \phi\right)+V(\phi)\right\}
\end{aligned}
$$


and this form leads to a real proper "time" everywhere

$$
d \tau=\sqrt{|N|} d t
$$

so that $n^{\alpha}=d x^{\alpha} / d \tau=(1 / \sqrt{|N|}, 0,0,0)$ is real and genuinely spacelike $n^{\alpha} \hat{n}_{\alpha}=+1$ in the Euclidean region. The momenta now stay real

$$
\begin{aligned}
\psi & =\frac{\phi^{\prime} a^{3} r^{2} \sin \theta}{\sqrt{|N|\left(1-k r^{2}\right)}} \\
K_{i j} & =\frac{3_{i j}^{\prime}}{\sqrt{|N|}} \\
p_{j}^{i} & =-\frac{2 a^{2} a^{\prime} r^{2} \sin \theta}{a \sqrt{|N|\left(1-k r^{2}\right)}} \delta_{j}^{i}
\end{aligned}
$$

and the squared momenta don't change sign,

$$
\begin{aligned}
\psi^{*} & =\frac{\left(\phi^{\prime}\right)^{2} a^{6} r^{4} \sin ^{2} \theta}{|N|\left(1-k r^{2}\right)} \\
\left(p^{*}\right)_{k}^{i} & =\frac{4\left(a^{\prime}\right)^{2} a^{4} r^{4} \sin ^{2} \theta}{|N|\left(1-k r^{2}\right)} \delta_{k}^{i}
\end{aligned}
$$

Thus the above requirements of smoothness and reality lead to the conditions that $\left(a^{\prime}\right)^{2} /|N|$ and $\left(\phi^{\prime}\right)^{2} /|N|$ be smooth, in agreement with the real "time" Darmois based approach.

\section{The Darmois approach with imaginary "time"}

We now apply the Darmois based approach to the quantum cosmology inspired model of signature change with an imaginary proper "time" in the Euclidean region, using the same metric. We list quantities that are different from above.

$$
\begin{aligned}
d \sigma & =\sqrt{N} d t=\sqrt{-\epsilon} d \tau \\
\hat{n}^{\alpha} & =\left(\frac{1}{\sqrt{N}}, 0,0,0\right), \quad \hat{n}_{\alpha} \hat{n}^{\alpha}=-1 \\
\hat{l}_{\alpha} & =(\sqrt{N}, 0,0,0), \quad \hat{l}_{\alpha} \hat{l}^{\alpha}=-1 \\
K_{i j} & =\frac{a a^{\prime}}{\sqrt{N}} \operatorname{diag}\left(\frac{1}{\left(1-k r^{2}\right)}, r^{2}, r^{2} \sin ^{2} \theta\right) \\
K & =\frac{3 a^{\prime}}{a \sqrt{N}}=3\left(\frac{d(\ln a)}{d \sigma}\right) \\
\tilde{\tilde{T}}^{00}=T^{\mu \nu} \hat{l}_{\mu} \hat{l}_{\nu} & =\frac{1}{2}\left\{\frac{\left(\phi^{\prime}\right)^{2}}{N}+V\right\}=\frac{1}{2}\left\{\left(\frac{d \phi}{d \sigma}\right)^{2}+V\right\} \\
\tilde{\tilde{G}}^{00}=G^{\mu \nu} \hat{l}_{\mu} \hat{l}_{\nu} & =\frac{3}{a^{2}}\left\{k+\frac{\left(a^{\prime}\right)^{2}}{N}\right\}=\frac{3}{a^{2}}\left\{k+\left(\frac{d a}{d \sigma}\right)^{2}\right\}
\end{aligned}
$$

where the double tilde means that the geodesic normal proper "time" coordinate $\sigma$ is imaginary where $N<0$. Applying the Darmois junction conditions as before, and again requiring $a, \phi$, 
$a^{\prime}$ and $\phi^{\prime}$ to be real, now gives

$$
[k]=0, \quad[a]=0, \quad\left[\frac{d a}{d \sigma}\right]=0
$$

which leads to

$$
k^{+}=k^{-}, \quad a_{\Sigma}^{+}=a_{\Sigma}^{-},\left.\quad i \frac{d a}{d \tau}\right|_{\Sigma} ^{+}=\left.\frac{d a}{d \tau}\right|_{\Sigma} ^{-}=0=\left.\frac{a^{\prime}}{\sqrt{N}}\right|_{\Sigma}
$$

by the usual argument [18]. The supplementary scalar field junction conditions

$$
[\phi]=0, \quad\left[\hat{n}^{\alpha} \partial_{\alpha} \phi\right]=\left[\frac{d \phi}{d \sigma}\right]=0, \quad[V]=0, \quad\left[\frac{d V}{d \phi}\right]=0
$$

lead to

$$
\phi_{\Sigma}^{+}=\phi_{\Sigma}^{-}, \quad V_{\Sigma}^{+}=V_{\Sigma}^{-},\left.\quad \frac{d V}{d \phi}\right|_{\Sigma} ^{+}=\left.\frac{d V}{d \phi}\right|_{\Sigma} ^{-},\left.\quad i \frac{d \phi}{d \tau}\right|_{\Sigma} ^{+}=\left.\frac{d \phi}{d \tau}\right|_{\Sigma} ^{-}=0=\left.\frac{\phi^{\prime}}{\sqrt{N}}\right|_{\Sigma}
$$

Clearly $\phi^{\prime}$ and $a^{\prime}$ must go to zero faster than $\sqrt{N}$. These requirements, derived using the imaginary Darmois approach, are in agreement with those of the imaginary Lagrangian approach and with quantum cosmological type signature change. This approach guarantees that $G_{\mu \nu}$ and $T_{\mu \nu}$ are smooth and finite, and conservation is observed, since the surface effects are all zero too

$$
E\left(\hat{l}_{\alpha}\right)=\frac{3}{a_{\Sigma}^{2}}\left[\frac{\left(a^{\prime}\right)^{2}}{N}\right]=\frac{1}{2}\left[\frac{\left(\phi^{\prime}\right)^{2}}{N}\right]=0, \quad E\left(\bar{e}_{\alpha}^{1}\right)=0, \quad E\left(\hat{n}^{\alpha}\right)=-E\left(\hat{l}_{\alpha}\right)=0, \quad E\left(\bar{e}_{1}^{\alpha}\right)=0
$$

Of course $G^{t t}$ and $T^{t t}$ are still divergent.

The EFEs with real "time"

When the EFEs (2)-(3) are calculated from (1), it is not necessary to specify the real or imaginary nature of the "time" direction, nor is it evident upon inspection. This is because a smooth signature changing metric does not contain information on whether tangent vectors to geodesics in the "time" direction change character across $\Sigma$.

In order to implement strictly classical signature change, we must include the fact that $n^{\alpha}$ changes from a (real) space-like direction to a (real) time-like direction; $n^{\mu} g_{\mu \nu} n^{\nu}=-\varepsilon$. Taking the derivative of this along $n^{\alpha}$ gives [5]

$$
n^{\mu} n^{\nu} n^{\lambda} \nabla_{\lambda} g_{\mu \nu}+2 n^{\mu} g_{\mu \nu} a^{\nu}=2 \delta(\tau), \quad a^{\nu}=n^{\lambda} \nabla_{\lambda} n^{\nu}
$$

So on $\Sigma$, either $a^{\nu}$ is not orthogonal to $n^{\nu}$, or $\nabla_{\lambda} g_{\mu \nu}$ is not zero, and the latter seems more likely given that signature change is a metric phenomenon. In any case, this introduces an extra degree of discontinuity not revealed by calculating the Einstein equations directly from the metric, so that we have to write down the results on either side of $\Sigma$, and then carefully examine the junction. The fundamental role played by the unit normal was stressed in $[2,6]$. Also, because of the metric degeneracy, a classical relativist suspects the $t$ coordinate may be badly behaved, just as the Schwarzschild time $T$ is, in the exterior Schwarzschild metric at 
$R=2 M$. Thus, with the EFEs (2)-(3) calculated directly from the metric (1) we project into $n^{\alpha}$ 's frame, obtaining

$$
\begin{gathered}
\tilde{G}_{0}^{0}=G_{\beta}^{\alpha} l_{\alpha} n^{\beta}=\kappa T_{\beta}^{\alpha} l_{\alpha} n^{\beta} \rightarrow \frac{3}{a^{2}}\left\{-k-\frac{\left(a^{\prime}\right)^{2}}{N}\right\}=\frac{\kappa}{2}\left\{-\frac{\left(\phi^{\prime}\right)^{2}}{N}-V\right\} \\
\varepsilon^{2} \tilde{G}_{00}=\tilde{G}^{00}=G^{\alpha \beta} l_{\alpha} l_{\beta}=\kappa T^{\alpha \beta} l_{\alpha} l_{\beta} \rightarrow \frac{3}{a^{2}}\left\{(\varepsilon) k+\frac{(\varepsilon)\left(a^{\prime}\right)^{2}}{N}\right\}=\frac{\kappa}{2}\left\{\frac{(\varepsilon)\left(\phi^{\prime}\right)^{2}}{N}+(\varepsilon) V\right\}
\end{gathered}
$$

Assuming that the values of $k, a, a^{\prime}, N, N^{\prime}, \phi^{\prime}$, and $V$ are continuous through $\Sigma$ suggests the signature change conditions (23) in order that the Einstein equations hold on both sides of $\Sigma$. However, going from Lorentzian $(\varepsilon=+1, N>0)$ to Euclidean $(\varepsilon=-1, N<0)$ regions, $\tilde{G}_{00}$, $\tilde{T}_{00}, \tilde{G}^{00}$, and $\tilde{T}^{00}$ clearly jump by $-\left(6 k / a_{\Sigma}^{2}\right)=-\kappa V_{\Sigma}$ - as in (19) — and this jump cannot be removed for $k \neq 0$. Since the quantities in (68) are all finite (unless $\Sigma$ is already singular in $M^{ \pm}$), and since the sign of $N$ changes across $\Sigma$, it follows that $\tilde{G}_{0}^{0}$, and $\tilde{T}_{0}^{0}$ also jump by $6(d(\ln a) / d \tau)_{\Sigma}^{2}=\kappa(d \phi / d \tau)_{\Sigma}^{2}$ - as in (21).

Similarly, projecting the momenta (39) and (42) into the "time"-like frame defined by $n^{\alpha}$, gives us (50) and (52) again.

\section{CONCLUSION}

We have just demonstrated that (a) the results of the strictly classical Darmois approach to signature change remain the same when squared-lapse coordinates are used; (b) when the Darmois approach is applied to the imaginary "time" model of signature change, it leads to Hayward's results; (c) when the Lagrangian approach is applied to the strictly classical model of signature change, our results are recovered.

There is a basic conceptual problem with this imaginary "time". Coordinate invariance and local Lorentz invariance are highly desireable features of classical Relativity. Can they be shown to hold in general for a metric structure with positive definite signature that admits vectors with negative magnitudes, in some physically meaningful sense? Are the Einstein equations well motivated? How do we know what is 'physically meaningful' in spaces with these properties? These issues need scrutiny. An interesting possible approach has been given in [22-23], in which the "time" direction is complex, and the Wick angle is treated as a dynamical degree of freedom.

Clearly the difference in results is entirely due to a different philosophy of signature change, and not at all due to the different approaches to junction conditions, or to the choice of coordinates. Strictly classical signature change does produce surface terms in the conservation law, but the imaginary time signature change has a Euclidean region with time-like features.

Hayward's approach focusses on maximising the smoothness of the covariant metric, the covariant Einstein tensor and the matter fields through $\Sigma$ in a particular type of coordinate system. Our approach focusses on the smoothness of the geometry and what kind of jumps in physical measurables, i.e. scalar invariants, are allowed across $\Sigma$. In his Lagrangian approach, Hayward effectively chooses imaginary "time", and his method then obtains well-defined rescaled EFEs through the signature change, at the cost of not-well-defined $G_{\mu \nu}$ and $T_{\mu \nu}$ and divergent $g^{\mu \nu}, G^{\mu \nu}$ and $T^{\mu \nu}$. We (Hellaby and Dray) choose real "time" and our method obtains well-defined, finite, coordinate-invariant expressions for the jumps in certain physical quantities at the signature change surface, at the cost of finite but discontinuous $g_{\mu \nu}$ and $g^{\mu \nu}$. Even in the absence of surface terms, bounded jumps in $G_{\mu \nu}$ and $T_{\mu \nu}$ are standard at boundaries. 
We leave the reader to weigh the two philosophies of signature change, and the problems associated with each.

The EFEs for a step function metric are badly defined. There is (as yet) no unique prescription for obtaining well defined distributional field equations. Hayward's postulated form does lead to his results, but requires a re-scaling of the EFEs. We postulate a form that doesn't require re-scaling and leads to our results. In the absence of a derivation of the distributional, signature-changing EFEs from first principles, one should be careful not to claim that a particular form of these equations is "the" EFEs. Rather, one must investigate and compare the properties of alternative definitions. We emphasize that in addition to being unrescaled, (33) and (34) are equivalent to (2) and (3) for continuous $N$, whereas the distributional form of $N^{2} \times(3)$ contains an extra distributional term that is not removed by the associated coordinate tranformation.

We regard Hayward's approaches to signature change as reasonable and interesting, but not the only possibilities.

\section{ACKNOWLEDGEMENTS}

We wish to thank George Ellis and David Coule for important comments. CH would like to thank the FRD for a research grant. TD was partially funded by NSF grant PHY-9208494.

\section{REFERENCES}

[1] C. Hellaby and T. Dray, Phys. Rev. D 49, 5096-5104 (1994).

[2] T. Dray and C. Hellaby, J. Math. Phys., to appear.

[3] G.F.R. Ellis, A. Sumeruk, D. Coule, and C. Hellaby, Class. Q. Grav. 9, 1535-54 (1992).

[4] G.F.R. Ellis, Gen. Rel. Grav. 24, 1047-68 (1992).

[5] G.F.R. Ellis and K. Piotrkowska, Int. J. Mod. Phys. D 3, 49 (1994).

[6] M. Carfora and G.F.R. Ellis, "The Geometry of Classical Change of Signature", SISSA report (unpublished), to appear in Int. J. Mod. Phys. D

[7] A. Sumeruk, C. Hellaby, and G.F.R. Ellis, University of Cape Town report (unpublished).

[8] T. Dray, C.A. Manogue, and R.W. Tucker, Gen. Rel. Grav. 23, 967-71 (1991).

[9] T. Dray, C.A. Manogue, and R.W. Tucker, Phys. Rev. D 48, 2587-90 (1993).

[10] T. Dray, C.A.Manogue, and R.W. Tucker, "The Effect of Signature Change on Scalar Field Propagation", Oregon State University report (Unpublished) (1993).

[11] T. Dray, C.A.Manogue, and R.W. Tucker, "Uniquness in the Presence of Signature Change", Lancaster University report (Unpublished) (1994).

[12] S.A. Hayward, Class. Q. Grav. 9, 1851-62 (1992); erratum ibid 92453 (1992).

[13] S.A. Hayward, Class. Q. Grav. 10, L7-11 (1993).

[14] S.A. Hayward, Phys. Rev. D 52, 7331-2 (1995).

[15] M. Kossowski and M. Kriele, Class. Q. Grav. 10, 1157-64 (1993). 
[16] M. Kossowski and M. Kriele, Class. Q. Grav. 10, 2363-71 (1993).

[17] J.J. Halliwell and J.B. Hartle, Phys. Rev. D 41, 1815-34 (1990).

[18] G.W. Gibbons and J.B. Hartle, Phys. Rev. D 42, 2458-68 (1990).

[20] R. Kerner and J. Martin, Class. Q. Grav. 10, 2111-22 (1993).

[21] J. Martin, Phys. Rev. D 49, 5086-95 (1994).

[22] J. Greensite, Phys. Lett. B 300, 34-7 (1993).

[23] E. Elizalde, S.D. Odintsov, and A. Romeo, Class. Q. Grav. 11, L61-7 (1994).

[24] Many results shown here were calculated using "G.R.Tensor" by K. Lake and P. Musgrave (Physics Department, Queen's University, Kingston, Ontario, CANADA; E-mail: grtensor@astro.queensu.ca), running under Maple V release 2.

[26] R.W. Tucker, T. Dray, D. Hartley, C.A. Manogue, and P. Tuckey, Tensor Distributions in the Presence of Degenerate Metrics, in preparation.

[27] C.Hellaby Solutions to Signature-Changing Field Equations, in preparation. 